\title{
Spectral frequency distribution for liquid deuterium determined by neutron scattering
}

\author{
M. Mukherjee and F. J. Bermejo \\ Instituto de Estructura de la Materia, Consejo Superior de Investigaciones Científicas, Serrano 123, Madrid E-28006, Spain \\ S. M. Bennington \\ Rutherford Appleton Laboratory, Chilton, Didcot, Oxon OX11 0QX, United Kingdom \\ B. Fåk \\ Département de Recherche Fondamentale sur la Matière Condensée, SPSMS/MDN, CEA Grenoble, 38054 Grenoble, France
}

(Received 26 January 1998; revised manuscript received 23 February 1998)

\begin{abstract}
The generalized spectral distribution, which is the frequency-domain representation of the velocity autocorrelation function, is determined for liquid molecular deuterium for a broad range of frequencies from neutronscattering experiments. Two well-defined features centered at frequencies of 3.8 and $7.6 \mathrm{meV}$ are seen superimposed on a broad, slowly decaying tail. The latter seems reminiscent of what has been observed for hydrogen in the dense-gas phase whereas the former is shown to arise from finite-frequency collective excitations and quantized molecular rotations. [S0163-1829(98)50718-7]
\end{abstract}

Although the generalized frequency distribution $Z(\omega)$ or its time-domain representation, the velocity autocorrelation function $\langle\mathbf{v}(0) \cdot \mathbf{v}(t)\rangle$, is perhaps the most relevant quantity to characterize the dynamics of any disordered material, explicit experimental results for simple liquids or dense gases are scarce. This is due to the difficulty of measuring the dynamic structure factor $S(Q, \omega)$ at rather low wave vectors, and to the absence of any widely accepted procedure to extrapolate data taken at finite $Q-\omega$ values to $Q=0$. In fact, accurate estimations of the velocity autocorrelation function of simple liquids from experiment are restricted to dense hydrogen gas ${ }^{1}$ and liquid alkali metals, ${ }^{2-4}$ although a number of results of lesser accuracy have been reported in several studies. ${ }^{5,6}$ Computer molecular dynamics has also been valuable in cases where the interaction potential is simple enough, such as liquid rare gases, the case of liquid Ar being paramount. ${ }^{7}$ The evaluation of the frequency distribution thus involves the extrapolation of the single-particle dynamic structure factor $S_{s}(Q, \omega)$ to zero wave vectors, ${ }^{2,8}$ which for a classical liquid becomes $Z(\omega)=z(Q \rightarrow 0, \omega)$, where

$$
z(Q, \omega)=\left(\frac{\omega^{2}}{Q^{2}}+A(Q)\right) S_{s}(Q, \omega)
$$

The function $A(Q)$ was introduced as an addition $^{2}$ to the Egelstaff formula ${ }^{8}$ to provide a more reliable representation of the low-frequency part of the spectrum, which is dominated by mass-diffusion effects. It amounts to a correction given by $D Q^{2}$, a hydrodynamic estimate for the spectral width in terms of the self-diffusion coefficient. For a liquid showing quantum effects to first order in $\hbar$, the singleparticle structure factor is given by the symmetrized quantity $\exp \left(-\frac{1}{2} \hbar \omega \beta\right) S_{s}(Q, \omega)$ with $\beta=\left(k_{B} T\right)^{-1}$.

The expression written above immediately evidences a singularity at $\omega \rightarrow 0$ and $Q \rightarrow 0$, which poses stringent conditions if very low- $\omega$ (below $0.25 \mathrm{meV}$ ) data are required. This was the case in investigations focused on the quest for the low- $\omega$ cusp arising from a long-time tail as predicted by kinetic theory formulations. An additional source of complication arises for systems where the coherent neutronscattering cross section is large. The coherent effects have here to be removed from the measured structure factor by means of appropriate angular averages or through modeling and subsequent subtraction. ${ }^{3}$

Here, our aim is focused on the dense-liquid phase of molecular deuterium under conditions close to freezing where collective effects are clearly evidenced ${ }^{9}$ and, contrary to the high-temperature gas, ${ }^{1}$ rotational motions become fully quantized. The interest in carrying out such a study is twofold. First, the frequency distribution for such a liquid is expected to be significantly different from that of the gas phase, where the most marked characteristic is a strong rise with decreasing frequency up to a finite intercept of $Z(\omega$ $=0)=D / \pi$ given by the macroscopic self-diffusion coefficient. It is also expected to exhibit significant differences with that for heavier diatomic liquids, where molecular rotations are describable by continuous diffusion treatments. Second, to explore up to what extent the behavior found in the gas phase, which is at present understood in terms of mode coupling (low- $\omega$ ) and Enskog (large- $\omega$ ) contributions, is still significant within the liquid phase. On the other hand, at temperatures of about $T=20 \mathrm{~K}$, quantum effects become relatively important for molecular deuterium. Recent results 9 have shown that the behavior of single-particle excitations in liquid $\mathrm{D}_{2}$ approaches an ideal-fluid regime for $Q$ $>4.2 \AA^{-1}$, while the low- $Q$ spectra $\left(Q \leqslant 1 \AA^{-1}\right)$ show a remarkably well defined phonon dispersion giving clear indications of strong anomalous dispersion, which is governed by phonon decay processes. In consequence, our aim is to explore the possibility of identifying within $Z(\omega)$ the frequency ranges that correspond to those found in our previous study: a phonon propagation regime for $Q<1.1 \AA^{-1}$ and $\omega$ $<9 \mathrm{meV}$, a classical-fluid regime at large wave vectors as discussed above, and an intermediate region, $1.8 \AA^{-1} \leqslant Q$ $\leqslant 2.4 \AA^{-1}$ which comprises the "roton" minimum of the dispersion curve. 
For a liquid composed by light particles one expects to find at least two different limiting behaviors in $S(Q, \omega)$. At low wave vectors $\left(0.3 \AA^{-1} \leqslant Q \leqslant 1.0 \AA^{-1}\right)$ the coherent response will be dominated by a quasielastic component ${ }^{10}$ plus a weaker phononlike excitation at some finite frequency well above the recoil energy $E_{r}=\hbar^{2} Q^{2} / 2 M$ (the effective mass $M$ can in principle be identified with the molecular mass). The $Q$ dependence of the intensity of these coherent effects mimics the static structure factor $S(Q)$, and the single-particle response, such as mass (self-) diffusion and molecular rotations, is masked. The rotational excitations give rise to narrow peaks at frequencies corresponding to $E_{K K^{\prime}}+E_{r}$, where $E_{K K^{\prime}} \approx B K\left(K^{\prime}+1\right)$ is the rotational level spacing, with $B$ $=3.8 \mathrm{meV}$. For larger momentum transfers, the excitations that matter are of single-particle nature since the coherent effects die away above $5 \AA^{-1} .^{11}$

The measurements reported here were carried out using the MARI chopper spectrometer at ISIS pulsed neutron source, where two instrumental configurations with incident energies of $E_{i}=10 \mathrm{meV}$ and $E_{i}=40 \mathrm{meV}$ were chosen to cover the low- and high-frequency regimes discussed above. The sample which was made of liquefied normal deuterium was held in a cylindrical container. $S(Q, \omega)$ was obtained from the measured cross section using well established procedures. ${ }^{9}$ The equilibrium composition of molecular deuterium at $20 \mathrm{~K}$ corresponds to a mixture of $97.8 \%$ ( $o$ ) ortho and $2.2 \%(p)$ para species. This results in a total cross section having a dominantly coherent component, although incoherent scattering arising mostly from $p \mathrm{D}_{2}$ will dominate the cross section for $Q \ll 1 \AA^{-1}$. 12

To determine $Z(\omega)$ from the experimental data, two paths have been followed. The first makes use of the relative advantage of liquid $\mathrm{D}_{2}$ that the scattering pattern at low wave vectors is strongly dominated by incoherent scattering arising from mostly para $\mathrm{D}_{2}$. This can be pictorially seen from the $S(\theta, E)$ surface plot shown in some of our previous communications. ${ }^{9}$ In fact, rather accurate bounds to the coherent and incoherent components can be obtained from a previous high-resolution study of liquid $\mathrm{D}_{2}$. An analysis ${ }^{9}$ of the quasielastic region of the spectra showed that (a) the hydrodynamic behavior (Fick's Law) was confirmed by the $Q^{2}$ dependence of the incoherent width concomitantly with the $Q^{-2}$ decrease of the peak amplitude for $Q \leqslant 1.6 \AA^{-1}$, (b) for $0.4 \leqslant Q \leqslant 1.6 \AA^{-1}$, the ratio $S_{i n c}(Q, 0) / S_{c o h}(Q, 0)$ varies from 750 to 40 , and (c) both the incoherent linewidth and peak intensity have the same proportionality constant (selfdiffusion coefficient).

The next step involved the computation of quantities given by Eq. (1) and extrapolation towards $Q \rightarrow 0$. Here, and contrary to previous derivations, straightforward use of a simple diffusion formula (Fick's law) is neither convenient nor adequate. As a matter of fact, a single spectral component does not seem to constitute an optimal recipe to extrapolate a complicated scattering law towards the hydrodynamic regime, since both quantized ortho-para transitions and additional finite-frequency excitations are involved. Since the very low-frequency end of the spectrum ( $\omega$ $<0.25 \mathrm{meV}$ ) was not of our immediate interest, the extrapolation was performed after rearranging $z(Q, \omega)$ into constant- $\omega$ slices and data for a given frequency was smoothly extrapolated towards $Q \rightarrow 0$. This procedure, also

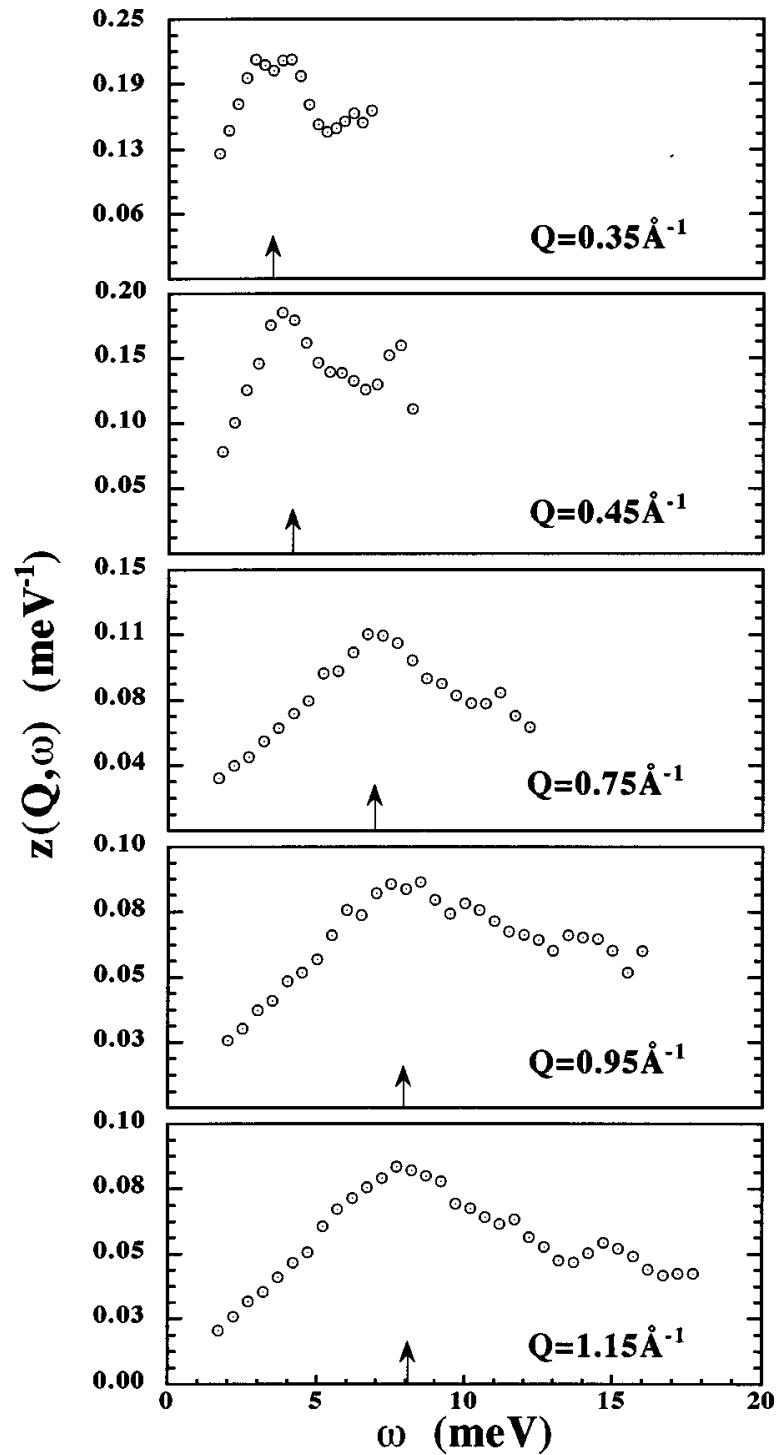

FIG. 1. Constant- $Q$ cuts of $z(Q, \omega)$ used in the extrapolations for values of the wave vector given as insets. The arrows indicate the excitation energies found in our previous analysis of the total dynamic structure factor.

used by other authors, ${ }^{4,13}$ has been shown to be robust enough, with exception made for frequencies comparable to the instrumental resolution.

The high- $Q$ data from the present experiment provide an alternative way to determine $Z(\omega)$ for higher frequencies, outside the hydrodynamic regime. To normalize the spectrum, the constant- $Q$ cuts were integrated over energy transfers so that the resulting $S(Q)=\int d \omega S(Q, \omega)$ was compared with equivalent magnitudes measured by neutron diffraction. $^{11}$

Figure 1 shows a set of $z(Q, \omega)$ functions for some selected wave vectors, calculated from the experimental data. The comparison between these data and those reported for dense $\mathrm{H}_{2}$ gas ${ }^{1}$ reveals the presence of a far richer structure in the liquid data. The sharp peak arising from the $J=0 \rightarrow 1$ rotational transition at about $7.6 \mathrm{meV}$ becomes visible in some of the low- $Q$ cuts, as well as a broad but well defined feature showing maxima between $3 \mathrm{meV}$ at the lowest $Q$ values and about $8 \mathrm{meV}$ at the highest momentum transfer 


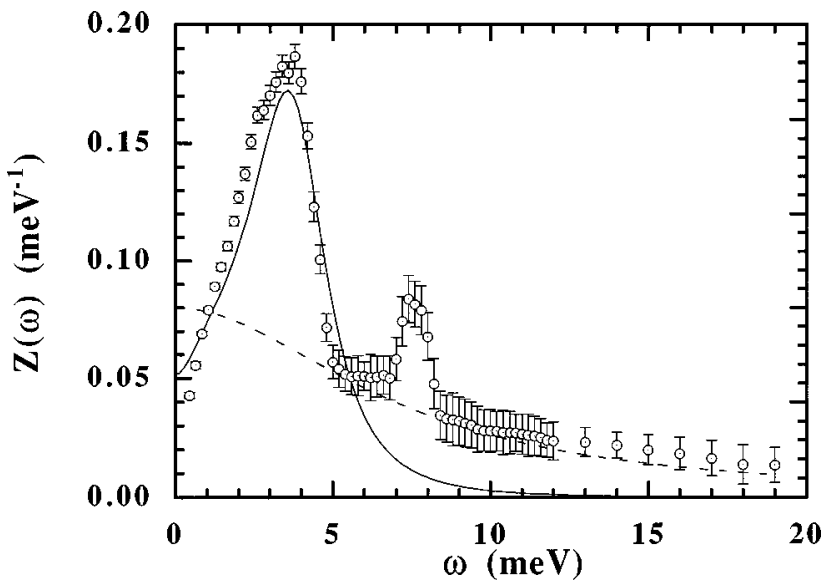

FIG. 2. The derived $Z(\omega)$ spectral frequency distribution (symbols). The solid line depicts a Brownian approximation for an Einstein oscillator with frequency $3.8 \mathrm{meV}$, number density $\rho=$ $0.023 \AA^{-3}$, Lennard-Jones parameters $\epsilon=36.7 \mathrm{~K}, \sigma=2.96 \AA$, a mass of 4 amu and the same thermodynamic constants as those of liquid $\mathrm{D}_{2}$. The dashed line gives an Enskog approximation to the high-frequency wing calculated using the same average time between collisions as for dense hydrogen gas.

plotted there. In terms of functions such as $z(Q, \omega)$, the spectra for $Q$ values above $Q_{p} \approx 2.1 \AA^{-1}$ [which corresponds to the maximum in $S(Q)$ ], are dominated by recoil broadened rotational peaks, leading to a broad maximum centered at 6-9 $\mathrm{meV}$ arising from a recoil shifted (and recoil broadened) $J=0 \rightarrow 1$ transition, a second peak at higher frequencies denoting the presence of higher-order rotational transitions, such as the $J=0 \rightarrow 2$ excitation $[B K(K+1)$ $=22.8 \mathrm{meV}],{ }^{14}$ and a small feature at about $15 \mathrm{meV}$, the origin of which could not be ascertained.

It is interesting to note that the frequencies corresponding to the main peaks appearing in $z(Q, \omega)$ are very close to the renormalized frequency ${ }^{15}$ (see Fig. 1) $\Omega_{Q}=\left(\omega_{Q}^{2}+\Gamma_{Q}^{2}\right)^{1 / 2}$ obtained from fitting a damped harmonic oscillator model to $S(Q, \omega)$, as was done in Ref. 9. The advantage with the present method is that it does not involve any model fitting. Furthermore, the frequencies $\Omega_{Q}$ are exactly the same as those of the maxima of $\omega^{2} S(Q, \omega)$. This lends additional support to the interpretation of the $\Omega_{Q}$ as the relevant physical frequency to describe the collective, damped harmonic motion in a liquid, rather than its "bare" (i.e., interactionfree) counterpart $\omega_{Q}$.

Figure 2 shows the spectral frequency distribution $Z(\omega)$ obtained from the measured $z(Q, \omega)$. Two well defined peaks centered at 3.8 and $7.6 \mathrm{meV}$ with a slowly decaying tail are observed. The first peak is the signature of the finitefrequency collective density oscillation whereas the second sharp peak very close to the theoretical value $(7.6 \mathrm{meV})$ of the $J=0 \rightarrow 1$ rotational transition is clearly due to the quantized molecular rotation. The relative amplitudes of the two finite-frequency peaks serve to quantify the importance of the rotational effects versus the coherent response in liquid deuterium. The former are expected to be far larger in liquid hydrogen, because of the substantially enhanced neutronscattering cross section for the para $\rightarrow$ orthohydrogen conversion.

As a consistency check, the first two frequency moments of $Z(\omega)$ have been evaluated and compared with the theoretical expectancies. The zeroth-frequency moment which equals $k_{B} T / M$ was found to be fulfilled within $\approx 1 \%$. The second-frequency moment was calculated from ${ }^{8}$

$$
\int_{0}^{\infty} d \omega \omega^{2} z(\omega)=\frac{\rho}{6 M^{2} \beta} \int d r g(r)\left[\frac{2}{r} \frac{\partial u(r)}{\partial r}+\frac{\partial^{2} u(r)}{\partial r^{2}}\right],
$$

where data for the intermolecular potential $u(r)$ and the static $g(r)$ radial distribution function were the same as used in the correlated-density-matrix studies reported in Ref. 9, and again, the fulfillment of the sum rule was verified up to a few percent.

The present estimate for the frequency spectrum of normal $\mathrm{D}_{2}$ at $20 \mathrm{~K}$ can be compared with that reported by Carneiro ${ }^{5}$ for liquid $\mathrm{H}_{2}$ at $14.7 \mathrm{~K}$. These liquids have very similar interaction potentials but their mass- and thermalwavelength effects are different. In liquid $\mathrm{H}_{2},{ }^{5} Z(\omega)$ shows two distinct contributions at energies of about 0.6 and 4.3 $\mathrm{meV}$, which are identified with mass diffusion and propagating excitations ("phonons"), respectively. Rotational excitations are expected at energies higher than those measured by Carneiro. In liquid $D_{2}, Z(\omega)$ is dominated by collective density excitations and the rotational transition. The former contribution to $Z(\omega)$ is in remarkable agreement with liquid $\mathrm{H}_{2}$. Diffusion effects are substantially reduced in $\mathrm{D}_{2}$, since the diffusion coefficient for deuterium at an equivalent thermodynamic state is approximately $30 \%$ below that of hydrogen. ${ }^{16}$

Finally, it seems worth comparing the frequency distribution for this liquid and that for liquid Ar as derived from molecular dynamics simulations. ${ }^{7}$ The shape of the $Z(\omega)$ curves for the two liquids is not too different, except for the rotational peak. The maximum which appears at $3.8 \mathrm{meV}$ in $\mathrm{D}_{2}$ is shifted down to $2 \mathrm{meV}$ in the simulated liquid Ar, and the ratio of its height to the value of $Z(\omega=0)$ is about 3.5 times higher in liquid $\mathrm{D}_{2}$, which is due to the larger lifetimes of the collective excitations in liquid $\mathrm{D}_{2}$. As in liquid $\mathrm{Ar}$, the low-frequency peak can be approximated by a model for the Brownian motion of a set of (monatomic) Einstein oscillators, ${ }^{17}$ using the oscillator frequency as an adjustable parameter, which in this case has to be close to the peak frequency. As also seen in Fig. 2, the agreement is reasonably good up to $5 \mathrm{meV}$. Well above the rotational peak (from $10 \mathrm{meV}$ onwards), the Brownian approximation severely breaks down. The shape of $Z(\omega)$ can be reproduced qualitatively using an Enskog formula like that employed for dense hydrogen gas, ${ }^{1}$ and quantitatively above $8 \mathrm{meV}$, retaining the Enskog average time between collisions, $\omega_{E} \approx 7.5 \mathrm{meV}$, used for the dense-gas phase and scaling the relevant diffusion coefficient.

This work was supported in part by DGICYT (Spain) Grant No. PB95-0075-c03-01. 
${ }^{1}$ P. Verkerk et al., Phys. Rev. A 40, 2860 (1989).

${ }^{2}$ C. Morkel and C. Gronemeyer, Z. Phys. B 72, 433 (1988).

${ }^{3}$ C. Morkel and W. Gläser, Phys. Rev. A 33, 3383 (1986); C. Morkel, C. Gronemeyer, W. Gläser, and J. Bosse, Phys. Rev. Lett. 58, 1873 (1987).

${ }^{4}$ T. Kinell et al., J. Phys. F 15, 1033 (1985); U. Dahlborg and L.G. Olsson, Phys. Rev. A 25, 2712 (1982).

${ }^{5}$ See K. Carneiro, Phys. Rev. A 14, 517 (1976), for liquid Ar at $T=85.2 \mathrm{~K}$ and liquid $\mathrm{H}_{2}$ at $T=14.7 \mathrm{~K}$.

${ }^{6}$ F. J. Bermejo et al., Phys. Rev. E 49, 3133 (1994).

${ }^{7}$ A. A. van Well, P. Verkerk, L. A. de Graaf, J. B. Suck, and J. R. D. Copley, Phys. Rev. A 31, 3391 (1985); C. Bruin, J. C. van Rijs, L. A. de Graaf, and I. M. de Schepper, ibid. 34, 3196 (1986). For an experimental estimate, see Carneiro (Ref. 5).

${ }^{8}$ P. A. Egelstaff and P. Schofield, Nucl. Sci. Eng. 12, 260 (1962). See also P. A. Egelstaff, Introduction to Liquid State Physics (Oxford Science Publications, Oxford, 1992), p. 243.

${ }^{9}$ F. J. Bermejo et al., Phys. Rev. B 47, 15097 (1993); M. Mukher- jee, F. J. Bermejo, B. Fåk, and S. M. Bennington, Europhys. Lett. 40, 153 (1997).

${ }^{10} \mathrm{~S}$. W. Lovesey, Theory of Neutron Scattering from Condensed Matter (Oxford Science Publications, Oxford, 1984), Vol. 1, p. 181.

${ }^{11}$ M. Zoppi et al., Phys. Rev. Lett. 75, 1779 (1995).

${ }^{12}$ F. J. Bermejo et al., Phys. Lett. A 158, 253 (1991).

${ }^{13}$ O. Söderström and U. Dahlborg, J. Phys. F 14, 2297 (1984).

${ }^{14}$ F. J. Mompean, M. Garcia, F. J. Bermejo, and S. M. Bennington, Phys. Rev. B 54, 970 (1996).

${ }^{15}$ H. R. Glyde, Excitations in Liquid and Solid Helium (Oxford Science Publications, Oxford, 1994), p. 187.

${ }^{16}$ H. M. Roder et al., Survey of the Properties of the Hydrogen Isotopes Below Their Critical Temperatures, NBS Technical Note 641 (U.S. Department of Commerce, Washington, DC, 1973).

${ }^{17}$ Calculation performed following formulae and arguments given in section 12.6 of Ref. 8. 